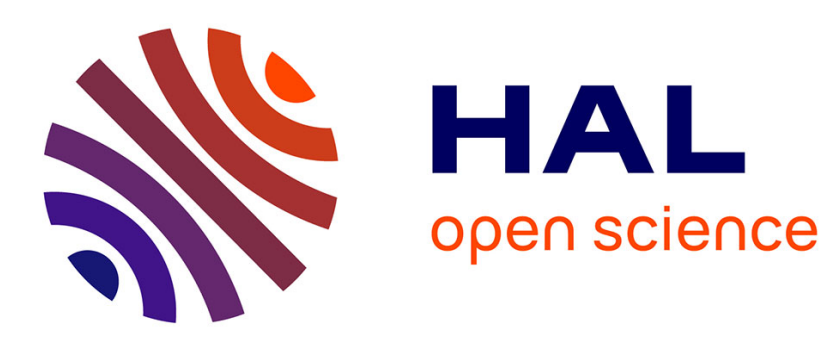

\title{
Stability of systems with the Duhem hysteresis operator: The dissipativity approach
}

\author{
Bayu Jayawardhana, Ruiyue Ouyang, Vincent Andrieu
}

\section{To cite this version:}

Bayu Jayawardhana, Ruiyue Ouyang, Vincent Andrieu. Stability of systems with the Duhem hysteresis operator: The dissipativity approach. Automatica, 2012, 48 (10), pp.2657-2662. 10.1016/j.automatica.2012.06.069 . hal-00754365

\section{HAL Id: hal-00754365 \\ https://hal.science/hal-00754365}

Submitted on 20 Nov 2012

HAL is a multi-disciplinary open access archive for the deposit and dissemination of scientific research documents, whether they are published or not. The documents may come from teaching and research institutions in France or abroad, or from public or private research centers.
L'archive ouverte pluridisciplinaire HAL, est destinée au dépôt et à la diffusion de documents scientifiques de niveau recherche, publiés ou non, émanant des établissements d'enseignement et de recherche français ou étrangers, des laboratoires publics ou privés. 


\title{
Stability of systems with Duhem hysteresis operator: Dissipativity approach *
}

\author{
Bayu Jayawardhana ${ }^{\mathrm{a}}$, Ruiyue Ouyang ${ }^{\mathrm{a}}$, Vincent Andrieu ${ }^{\mathrm{b}}$ \\ ${ }^{a}$ Dept. Discrete Technology and Production Automation, University of Groningen, Groningen 9747AG, The Netherlands \\ ${ }^{\mathrm{b}}$ Université Lyon 1, Villeurbanne; CNRS, UMR 5007, LAGEP. 43 bd du 11 novembre, 69100 Villeurbanne, France.
}

\begin{abstract}
In this paper, we discuss the dissipativity property of Duhem hysteresis operator. Under mild assumptions on the functions which define the Duhem operator, an explicit construction of the storage functions satisfying a dissipation inequality is given based on the data on these functions. The constructed storage function is also related to the underlying anhysteresis function which is commonly used to describe hysteresis in magnetic material. The results can thus facilitate analysis of systems with Duhem operator via dissipativity approach.
\end{abstract}

Key words: Hysteresis, dissipative systems, passive systems, nonlinear systems.

\section{Introduction}

Hysteresis is a nonlinear phenomenon that is commonly found in a wide range of physical systems, such as, magnetic material, piezo-electric material and mechanical friction. For studying the influence of hysteresis in a system, numerous hysteresis models have been proposed, such as the backlash, elastic-plastic, Preisach and Duhem models [3,15].

In the literature on magnetic materials, the hysteresis behavior is caused by the friction/pinning of the magnetic domain-walls $[12,20]$. When the influence of the friction/pinning of magnetic domain-walls is neglected, the relation between the magnetization and the external magnetic field defines the anhysteresis behavior. In this case, Jiles and Atherton [12] propose a hysteresis model for describing the magnetization that is composed of an anhysteresis part and another component which is due to the pinning of magnetic domain-walls. Similarly, Coleman and Hodgdon [4] propose another hysteresis model to describe the same phenomenon. These models are particular classes of Duhem model [15].

\footnotetext{
* This paper was not presented at any IFAC meeting. Corresponding author B. Jayawardhana. Tel. +31-50-3637156.

Email addresses: bayujw@ieee.org (Bayu

Jayawardhana), r.ouyang@rug.nl (Ruiyue Ouyang), vincent. andrieu@gmail. com (Vincent Andrieu).
}

Hysteresis phenomenon that is due to the friction, either between the magnetic domain-walls or between mechanical surfaces, dissipates energy by heat. This is related to the concept of dissipativity in the systems theory literature $[19,21]$, where the energy transfer can be described by constructing a storage function satisfying a certain dissipation inequality. Based on this concept, various stability analysis and controller designs for dissipative systems have been proposed, see, for example, $[10,17,19]$.

Consequently, the dissipative characterization of hysteresis operators can be useful for analyzing the stability of hysteretic systems and for designing a stabilizing controller. In Gorbet et al.[5], it is shown that for a Preisach hysteresis operator $\Phi$ with a non-negative weighting function, a positive semi-definite function $H: \mathbb{R}^{2} \rightarrow \mathbb{R}_{+}$ can be constructed such that for every continuous input signal $u$,

$$
\frac{\mathrm{d} H(y(t), u(t))}{\mathrm{d} t} \leq \dot{y}(t) u(t), \quad \forall t \geq 0,
$$

where the signal $y:=\Phi\left(u, y_{0}\right)$ and $y_{0}:=y(0)$ is the initial condition of the Preisach operator. This dissipativity property has been employed in [5] to show the stability of electro-mechanical systems with a hysteretic piezo-actuator. By exploiting the dissipativity property, the controller can be designed without having to determine an inverse model of the hysteresis which is difficult to construct but has been used to counteract the hys- 
teresis behavior (see, for example, [7]). For relay and backlash operators, the corresponding storage function has been proposed in Brokate and Sprekels [3].

However, the existence of such a function $H$ for the Duhem model is not well-known despite the applicability of Duhem model for describing hysteresis phenomena in magnetic materials, such as, the magnetic electron lenses [2]. A preliminary result for a small class of Duhem model has been presented in Jayawardhana and Andrieu [11]. In this paper, we extend the result in [11] by admitting a larger class of Duhem model that admits anhysteresis function (the precise definition of anhysteresis function is given in Section 3).

\section{Preliminaries}

For an open set $U \in \mathbb{R}^{n}$, we denote $C^{1}(U)$ the space of continuously differentiable functions $f: U \rightarrow \mathbb{R}$ and $A C(U)$ the space of absolutely continuous functions $f: U \rightarrow \mathbb{R}$. For any real valued function $z$, we denote $\dot{z}(t):=\frac{\mathrm{d}}{\mathrm{d} t} z(t)$ when it makes sense.

Using the same description as in $[15,16,22]$, the Duhem operator $\Phi: A C\left(\mathbb{R}_{+}\right) \times \mathbb{R} \rightarrow A C\left(\mathbb{R}_{+}\right),\left(u, y_{0}\right) \mapsto$ $\Phi\left(u, y_{0}\right)=: y$ is described by

$$
\dot{y}(t)=f_{1}(y(t), u(t)) \dot{u}_{+}(t)+f_{2}(y(t), u(t)) \dot{u}_{-}(t),
$$

where $y(0)=y_{0}, \dot{u}_{+}(t):=\max \{0, \dot{u}(t)\}, \dot{u}_{-}(t):=$ $\min \{0, \dot{u}(t)\}$ and $f_{1}, f_{2} \in C^{1}\left(\mathbb{R}^{2}\right)$.

The existence of solutions to (1) has been reviewed in [15]. In particular, if for every $v \in \mathbb{R}, f_{1}$ and $f_{2}$ satisfy

$\left(\gamma_{1}-\gamma_{2}\right)\left[f_{1}\left(\gamma_{1}, v\right)-f_{1}\left(\gamma_{2}, v\right)\right] \leq \lambda_{1}(v)\left(\gamma_{1}-\gamma_{2}\right)^{2}$,

$\left(\gamma_{1}-\gamma_{2}\right)\left[f_{2}\left(\gamma_{1}, v\right)-f_{2}\left(\gamma_{2}, v\right)\right] \geq-\lambda_{2}(v)\left(\gamma_{1}-\gamma_{2}\right)^{2}$,

for all $\gamma_{1}, \gamma_{2} \in \mathbb{R}$, where $\lambda_{1}$ and $\lambda_{2}$ are nonnegative, then the solution to (1) exist and $\Phi$ maps $A C\left(\mathbb{R}_{+}\right) \times \mathbb{R} \rightarrow A C\left(\mathbb{R}_{+}\right)$.

The Duhem operator in (1) encapsulates many different models which have been proposed in literature, such as, the Bouc-Wen model [8], the LuGre friction model [23], the Coleman-Hodgdon model [2,4], the Dahl model and the Jiles-Atherton model [12].

Definition 2.1 The Duhem operator as in (1) is said to be dissipative with respect to the supply rate $\dot{y} u$ if there exists a non-negative function $H: \mathbb{R}^{2} \rightarrow \mathbb{R}_{+}$such that for every $u \in A C\left(\mathbb{R}_{+}\right)$and $y_{0} \in \mathbb{R}$

$$
\frac{\mathrm{d} H(y(t), u(t))}{\mathrm{d} t} \leq \dot{y}(t) u(t)
$$

holds for almost all $t \in \mathbb{R}_{+}$with $y:=\Phi\left(u, y_{0}\right)$.

By integrating the dissipation inequality (2) from 0 to $T$, we have $H(y(T), u(T))-H(y(0), u(0)) \leq$ $\int_{0}^{T} \dot{y}(\tau) u(\tau) \mathrm{d} \tau$. Since $H \geq 0, \int_{0}^{T} \dot{y}(\tau) u(\tau) \mathrm{d} \tau \geq$
$-H(y(0), u(0))$ holds for all $T \geq 0$. By relating it to the work by Angeli [1], this inequality implies that such a Duhem operator satisfying (2) has a counter-clockwise input-output dynamics which, for linear systems, is equivalent to the negative-imaginary linear systems as discussed in [14]. We remark that the inequality (2) in the Definition 2.1 has also been used in the study of dissipativity for Preisach model [5] and semi-linear Duhem model [18]. This dissipation inequality belongs also to the family of generalized dissipation inequality as described in [21]. In this paper, the dissipation inequality of the Duhem operator is studied based on the solutions $(y, u)$ of the underlying differential equation (1).

\section{Main results}

In this section, we show that under mild assumptions on $f_{1}$ and $f_{2}$, we can obtain explicitly a family of storage functions $H$ which satisfies (2). It is constructed based only on the knowledge of $f_{1}$ and $f_{2}$.

Before we can state our main results in Theorem 3.3 and 3.4 given respectively in subsections 3.4 and 3.5 , we need to introduce three functions in the following subsections: an anhysteresis function $f_{a n}$, a traversing function $\omega_{\Phi}$ (or $\nu_{\Phi}$ ) and an intersecting function $\Omega$ (or $\Upsilon$ ). These functions are defined based on $f_{1}$ and $f_{2}$, and they play important roles in the construction of the storage function and in the characterization of dissipativity. Generally speaking, the anhysteresis function $f_{a n}$ defines the curve where $f_{1}=f_{2}$, the traversing function $\omega_{\Phi}$ describes the trajectory of $\Phi$ when a monotone increasing $u$ or a monotone decreasing $u$ is applied from a given initial condition, and the intersecting function $\Omega$ defines the intersection of $f_{a n}$ and $\omega_{\Phi}$ from a given initial condition. The dual interpretation is applicable to the traversing function $\nu_{\Phi}$ and its corresponding intersecting function $\Upsilon$.

\subsection{The anhysteresis function}

In order to define the anhysteresis function, we rewrite $f_{1}$ and $f_{2}$ as follows

$$
\left.\begin{array}{l}
f_{1}(\gamma, v)=F(\gamma, v)+G(\gamma, v), \\
f_{2}(\gamma, v)=-F(\gamma, v)+G(\gamma, v),
\end{array}\right\} \forall(\gamma, v) \in \mathbb{R}^{2} .
$$

where $F:=\frac{f_{1}-f_{2}}{2}, G:=\frac{f_{1}+f_{2}}{2}$. We assume that the implicit function $v \mapsto\{\gamma \in \mathbb{R}, F(\gamma, v)=0\}$ admits a unique explicit solution $\gamma=f_{a n}(v)$ (or $v=g_{a n}(\gamma)$ ) where $f_{a n}$ (or $g_{a n}$ ) is $C^{1}$. Such function $f_{a n}\left(\right.$ or $\left.g_{a n}\right)$ is called an anhysteresis function and the corresponding graph $\left\{\left(v, f_{a n}(v)\right) \mid v \in \mathbb{R}\right\}$ (or $\left\{\left(g_{a n}(\gamma), \gamma \mid \gamma \in \mathbb{R}\right\}\right)$ is called an anhysteresis curve. Using $f_{\text {an }}$, it can be checked that $f_{1}\left(f_{a n}(v), v\right)=f_{2}\left(f_{a n}(v), v\right)$ holds for all $v \in \mathbb{R}$.

The dissipativity property for Duhem model with $g_{a n} \equiv$ 0 has been presented in our preliminary work in [11]. 


\subsection{The traversing function}

For every pair $\left(y_{0}, u_{0}\right) \in \mathbb{R}^{2}$, let $\omega_{\Phi, 1}\left(\cdot, y_{0}, u_{0}\right)$ : $\left[u_{0}, \infty\right) \rightarrow \mathbb{R}$ be the solution $z$ of

$z(v)-y_{0}=\int_{u_{0}}^{v} f_{1}(z(\sigma), \sigma) \mathrm{d} \sigma$, for all $v \in\left[u_{0}, \infty\right)$ and let $\omega_{\Phi, 2}\left(\cdot, y_{0}, u_{0}\right):\left(-\infty, u_{0}\right] \rightarrow \mathbb{R}$ be the solution $z$ of $z(v)-y_{0}=\int_{u_{0}}^{v} f_{2}(z(\sigma), \sigma) \mathrm{d} \sigma$, for all $v \in\left(-\infty, u_{0}\right]$. Using the above definitions, for every pair $\left(y_{0}, u_{0}\right) \in \mathbb{R}^{2}$, the function $\omega_{\Phi}\left(\cdot, y_{0}, u_{0}\right): \mathbb{R} \rightarrow \mathbb{R}$ is defined by the concatenation of $\omega_{\Phi, 2}\left(\cdot, y_{0}, u_{0}\right)$ and $\omega_{\Phi, 1}\left(\cdot, y_{0}, u_{0}\right)$ :

$$
\omega_{\Phi}\left(v, y_{0}, u_{0}\right)=\left\{\begin{array}{l}
\omega_{\Phi, 2}\left(v, y_{0}, u_{0}\right) \forall v \in\left(-\infty, u_{0}\right) \\
\omega_{\Phi, 1}\left(v, y_{0}, u_{0}\right) \forall v \in\left[u_{0}, \infty\right) .
\end{array}\right.
$$

Similarly, we can introduce the function $\nu_{\Phi}$ which is dual to construction of $\omega_{\Phi}$. For every pair $\left(y_{0}, u_{0}\right) \in \mathbb{R}^{2}$, let $\nu_{\Phi, 1}\left(\cdot, y_{0}, u_{0}\right):\left[u_{0}, \infty\right) \rightarrow \mathbb{R}$ be the solution $z$ of $z(v)-y_{0}=\int_{u_{0}}^{v} f_{2}(z(\sigma), \sigma) \mathrm{d} \sigma$, for all $v \in\left[u_{0}, \infty\right)$ and let $\nu_{\Phi, 2}\left(\cdot, y_{0}, u_{0}\right):\left(-\infty, u_{0}\right] \rightarrow \mathbb{R}$ be the solution $z$ of $z(v)-y_{0}=\int_{u_{0}}^{v} f_{1}(z(\sigma), \sigma) \mathrm{d} \sigma$, for all $v \in\left(-\infty, u_{0}\right]$. Using the above definitions, for every pair $\left(y_{0}, u_{0}\right) \in \mathbb{R}^{2}$, the function $\nu_{\Phi}\left(\cdot, y_{0}, u_{0}\right): \mathbb{R} \rightarrow \mathbb{R}$ is defined by the concatenation of $\nu_{\Phi, 2}\left(\cdot, y_{0}, u_{0}\right)$ and $\nu_{\Phi, 1}\left(\cdot, y_{0}, u_{0}\right)$ :

$$
\nu_{\Phi}\left(v, y_{0}, u_{0}\right)=\left\{\begin{array}{l}
\nu_{\Phi, 2}\left(v, y_{0}, u_{0}\right) \forall v \in\left(-\infty, u_{0}\right) \\
\nu_{\Phi, 1}\left(v, y_{0}, u_{0}\right) \forall v \in\left[u_{0}, \infty\right) .
\end{array}\right.
$$

\subsection{Intersecting function}

As indicated before, the function $\Omega$ defines the intersection between $\omega_{\Phi}(\cdot, \gamma, v)$ and $f_{a n}(\cdot)$. More precisely, the function $\Omega: \mathbb{R}^{2} \rightarrow \mathbb{R}$ is an intersecting function (corresponding to $\omega_{\Phi}$ and $\left.f_{a n}\right)$ if $\omega_{\Phi}(\Omega(\gamma, v), \gamma, v)=$ $f_{a n}(\Omega(\gamma, v))$ for all $(\gamma, v) \in \mathbb{R}^{2}$; and $\Omega(\gamma, v) \geq v$ whenever $\gamma \geq f_{a n}(v)$ and $\Omega(\gamma, v)<v$ otherwise. This implies that the two functions $\omega_{\Phi}(\cdot, \gamma, v)$ and $f_{a n}(\cdot)$ intersect at a unique point larger or smaller than $u_{0}$ depending on the sign of $\gamma-f_{a n}(v)$. In our main result, we also need that $\frac{\mathrm{d} \Omega(y(t), u(t))}{\mathrm{d} t}$ exists for every solutions $(y, u)$ of $(1)$.

The following lemma gives sufficient conditions on $f_{1}$ and $f_{2}$ for the existence of such an intersecting function $\Omega$.

Lemma 3.1 [9, Lemma 3.1] Assume that $f_{1}$ and $f_{2}$ in (3) be such that $f_{1}, f_{2}$ and $f_{\text {an }}$ are $C^{1}$. Moreover, assume that $f_{a n}$ is strictly increasing and there exists a positive real number $\epsilon$ such that for all $(\gamma, v) \in \mathbb{R}^{2}$

$$
\begin{aligned}
& f_{1}(\gamma, v)<\frac{\mathrm{d} f_{a n}(v)}{\mathrm{d} v}-\epsilon \text { whenever } \gamma>f_{a n}(v) \text { and } \\
& f_{2}(\gamma, v)<\frac{\mathrm{d} f_{a n}(v)}{\mathrm{d} v}-\epsilon \text { whenever } \gamma<f_{a n}(v)
\end{aligned}
$$

hold. Then there exists an intersecting function $\Omega \in$ $C^{1}\left(\mathbb{R}^{2}\right)$ (corresponding to $\omega_{\Phi}$ and $f_{\text {an }}$ ) such that for all $u \in A C\left(\mathbb{R}_{+}\right), y_{0} \in \mathbb{R}$ and $y:=\Phi\left(u, y_{0}\right), \frac{\mathrm{d}}{\mathrm{d} t} \Omega(y(t), u(t))$ exists for almost all $t$.

For the proof of Lemma 3.1, we refer to [9, Lemma 3.1]. Similarly, we can define an intersecting function $\Upsilon$ describing the intersection between $\nu_{\Phi}(\cdot, \gamma, v)$ and $f_{a n}(\cdot)$. The function $\Upsilon: \mathbb{R}^{2} \rightarrow \mathbb{R}$ is an intersecting function (corresponding to $\nu_{\Phi}$ and $\left.f_{a n}\right)$ if $\nu_{\Phi}(\Upsilon(\gamma, v), \gamma, v)=$ $f_{\text {an }}(\Upsilon(\gamma, v))$ for all $(\gamma, v) \in \mathbb{R}^{2}$; and $\Upsilon(\gamma, v) \geq v$ whenever $\gamma \geq f_{a n}(v)$ and $\Upsilon(\gamma, v)<v$ otherwise. We also require that $\frac{\mathrm{d} \Upsilon(y(t), u(t))}{\mathrm{d} t}$ exists for every solutions $(y, u)$ of (1). The following lemma gives sufficient conditions on $f_{1}$ and $f_{2}$ for the existence of such an intersecting function $\Upsilon$.

Lemma 3.2 Assume that $f_{1}$ and $f_{2}$ in (3) be such that $f_{1}, f_{2}$ and $f_{\text {an }}$ are $C^{1}$. Moreover, assume that $f_{\text {an }}$ is strictly increasing and there exists a positive real number $\epsilon$ such that for all $(\gamma, v) \in \mathbb{R}^{2}$

$f_{2}(\gamma, v)<\frac{\mathrm{d} f_{a n}(v)}{\mathrm{d} v}-\epsilon$ whenever $\gamma>f_{a n}(v)$ and

$f_{1}(\gamma, v)<\frac{\mathrm{d} f_{a n}(v)}{\mathrm{d} v}-\epsilon$ whenever $\gamma<f_{a n}(v)$

hold. Then there exists an intersecting function $\Upsilon \in$ $C^{1}\left(\mathbb{R}^{2}\right)$ (corresponding to $\nu_{\Phi}$ and $f_{\text {an }}$ ) such that for all $u \in A C\left(\mathbb{R}_{+}\right), y_{0} \in \mathbb{R}$ and $y:=\Phi\left(u, y_{0}\right), \frac{\mathrm{d}}{\mathrm{d} t} \Upsilon(y(t), u(t))$ exists for almost all $t$.

\subsection{Storage function using $\omega_{\Phi}$}

Under the hypotheses in Lemma 3.1, we can define a candidate storage function $H_{1}: \mathbb{R}^{2} \rightarrow \mathbb{R}$ by

$H_{1}(\gamma, v)=\gamma v+\int_{v}^{\Omega(\gamma, v)} \omega_{\Phi}(\sigma, \gamma, v) \mathrm{d} \sigma-\int_{0}^{\Omega(\gamma, v)} f_{a n}(\sigma) \mathrm{d} \sigma$,

where $\Omega$ is the intersecting function (corresponding to $\omega_{\Phi}$ and $\left.f_{a n}\right)$.

Theorem 3.3 Consider the Duhem hysteresis operator $\Phi$ defined in (1)-(3) with $C^{1}$ functions $F, G: \mathbb{R}^{2} \rightarrow \mathbb{R}$ and the corresponding anhysteresis function $f_{\text {an }}$ be $C^{1}$. Suppose that there exists an intersecting function $\Omega$ and the following condition holds for all $(\gamma, v) \in \mathbb{R}^{2}$ (A) $F(\gamma, v) \geq 0$ whenever $\gamma \leq f_{a n}(v)$, and $F(\gamma, v)<0$
otherwise.

Then for every $u \in A C\left(\mathbb{R}_{+}\right)$and for every $y_{0} \in \mathbb{R}$, the function $t \mapsto H_{1}\left((y(t), u(t))\right.$ with $\left.y:=\Phi\left(u, y_{0}\right)\right)$ and $H_{1}$ as in (10) is differentiable and satisfies (2). Moreover, if $f_{1} \geq 0$ and $f_{2} \geq 0$ then $\Phi$ is dissipative with respect to 
the supply rate $\dot{y} u$ with the storage function $H_{1}$, i.e., $H_{1}$ is non-negative.

The proof can be found in the Appendix A.

\subsection{Storage function using $\nu_{\Phi}$}

Dual to the results from the previous subsection, under the hypotheses of Lemma 3.2 we can also define a storage function based on $\nu_{\Phi}$. The candidate storage function $H_{2}: \mathbb{R}^{2} \rightarrow \mathbb{R}$ is given by

$H_{2}(\gamma, v)=\gamma v+\int_{v}^{\Upsilon(\gamma, v)} \nu_{\Phi}(\sigma, \gamma, v) \mathrm{d} \sigma-\int_{0}^{\Upsilon(\gamma, v)} f_{a n}(\sigma) \mathrm{d} \sigma$

where $\Upsilon$ is the intersecting function (corresponding to $\nu_{\Phi}$ and $\left.f_{a n}\right)$.

Theorem 3.4 Consider the Duhem hysteresis operator $\Phi$ defined in (1)-(3) with $C^{1}$ functions $F, G: \mathbb{R}^{2} \rightarrow \mathbb{R}$ and the corresponding anhysteresis function $f_{\text {an }}$ be $C^{1}$. Suppose that there exists an intersecting function $\Upsilon$ and Assumption (A) in Theorem 3.3 hold. Then for every $u \in A C\left(\mathbb{R}_{+}\right)$and for every $y_{0} \in \mathbb{R}$, the function $t \mapsto$ $H_{2}\left((y(t), u(t))\right.$ with $\left.y:=\Phi\left(u, y_{0}\right)\right)$ and $H_{2}$ as in $(11)$ is differentiable and satisfies (2). Moreover, if $f_{1} \geq 0$ and $f_{2} \geq 0$ then $\Phi$ is dissipative with respect to the supply rate yu with the storage function $\mathrm{H}_{2}$, i.e., $\mathrm{H}_{2}$ is non-negative.

The proof is similar to the proof of Theorem 3.3.

Remark 3.5 If $f_{1}$ and $f_{2}$ satisfy the assumptions in both Theorem 3.3 and Theorem 3.4, the convex combination of $\mathrm{H}_{1}$ and $\mathrm{H}_{2}$ is also a storage function which satisfies (2). Moreover, if additionally, it is assumed that $f_{1}$ and $f_{2}$ are positive then the convex combination of $H_{1}$ and $\mathrm{H}_{2}$ is also a non-negative storage function.

\section{$4 \quad$ Stability analysis}

The functions $H_{1}$ and $H_{2}$ which are used in Theorem 3.3 and 3.4, respectively, are non-negative if both $f_{1}$ and $f_{2}$ are positive-definite. Moreover, from the definition of $H_{1}$ in (10), the null-set of $H_{1}$, i.e., $\left\{(\gamma, v) \in \mathbb{R}^{2} \mid H_{1}(\gamma, v)=\right.$ $0\}$ is given by $\mathbb{R} \times\{0\}$. This set is also the same as that of $\mathrm{H}_{2}$ in (11). Consider now an interconnected system with Duhem hysteresis operator $\Phi$ satisfying the hypotheses of Theorem 3.3 with positive-definite $f_{1}$ and $f_{2}$ as follows

$$
\dot{x}=f(x, y), \quad u=h(x, y), \quad y=\Phi\left(u, y_{0}\right),
$$

where $f, h$ are smooth functions with $f(0,0)=0$, $h(0,0)=0$ and $x \in \mathbb{R}^{n}$ is the state of the first subsystem.
Proposition 4.1 Assume that $f_{1}$ and $f_{2}$ satisfy

$$
\left|1-f_{1}(\gamma, v) \frac{\partial h}{\partial \gamma}(\xi, \gamma)\right| \geq \epsilon,\left|1-f_{2}(\gamma, v) \frac{\partial h}{\partial \gamma}(\xi, \gamma)\right| \geq \epsilon
$$

for all $(\xi, \gamma) \in \mathbb{R}^{n+1}$ where $v=h(\xi, \gamma)$ and $\epsilon>0$. Suppose that there exists a radially unbounded function $W: \mathbb{R}^{n+1} \rightarrow \mathbb{R}_{+}$for the first subsystem such that

$$
\frac{\partial W(\xi, \gamma)}{\partial \xi} f(\xi, \gamma)+\frac{\partial W(\xi, \gamma)}{\partial \gamma} w \leq-\alpha(\xi, \gamma)-h(\xi, \gamma) w
$$

for all $\xi \in \mathbb{R}^{n}$ and for all $\gamma, w \in \mathbb{R}$, where $\alpha$ is a positive semi-definite function. Then the solutions of the closed loop system $(x(t), y(t))$ converges to the set $\{(\xi, \gamma) \in$ $\left.\mathbb{R}^{n+1} \mid \alpha(\xi, \gamma)=0\right\}$.

Proof: Let $V(x, y)=W(x, y)+H_{1}(y, u)$ be the Lyapunov function for (12) where

$$
\begin{aligned}
\dot{V} & =\frac{\partial W(x, y)}{\partial x} f(x, y)+\frac{\partial W(x, y)}{\partial y} \dot{y}+\frac{\mathrm{d} H_{1}\left(\Phi\left(u, z_{0}\right), u\right)}{\mathrm{d} t} \\
& \leq-\alpha(x, y)-h(x, y) \dot{y}+\overbrace{\Phi\left(y, z_{0}\right)} u=-\alpha(x, y) .
\end{aligned}
$$

Since $V$ is lower-bounded, the solutions $(x(t), y(t))$ are bounded for all $t \geq 0$. With (12), it yields that the time function $x(t)$ is uniformly continuous. Moreover, for all $t$ such that $\dot{u}(t) \geq 0$, we have

$$
\dot{y}=f_{1}(y, u)\left[\frac{\partial h}{\partial x}(x, y) f(x, y)+\frac{\partial h}{\partial y}(x, y) \dot{y}\right] .
$$

Hence,

$\dot{y}\left[1-f_{1}(y, u) \frac{\partial h}{\partial y}(x, y)\right]=f_{1}(y, u) \frac{\partial h}{\partial x}(x, y) f(x, y)$.

Also, when $\dot{u}(t) \leq 0$, it yields

$\dot{y}\left[1-f_{2}(y, u) \frac{\partial h}{\partial y}(x, y)\right]=f_{2}(y, u) \frac{\partial h}{\partial x}(x, y) f(x, y)$.

Using (13) and using the fact that $(x, y)$ is bounded, we obtain that $y$ is a uniformly continuous function. Consequently, using the Barbalat's lemma, the solutions of the closed loop system $(x(t), y(t))$ converges to the set $\left\{(\xi, \gamma) \in \mathbb{R}^{n+1} \mid \alpha(\xi, \gamma)=0\right\}$.

\section{Conclusions}

In this paper, a class of Duhem model which is dissipative with respect to the supply rate $\dot{y} u$ has been identified and the corresponding family of storage functions has been presented. The class includes models that are widely-used to describe magnetic material, such as, the Jiles-Atherton and Coleman-Hodgdon models. Therefore the analysis that is presented in this paper is relevant to the stability analysis and control design for systems that use electromagnetic-based actuators, e.g., voice-coil actuators. A possible control design methodology that 
can exploit this dissipativity property is based on the interconnection of counterclockwise systems as discussed in [1] and [14].

\section{A Proof of Theorem 3.3}

It can be checked that the hypothesis (A) on $F$ implies that $f_{1}(\gamma, v) \geq f_{2}(\gamma, v)$ whenever $\gamma \leq f_{a n}(v)$, and $f_{1}(\gamma, v)<f_{2}(\gamma, v)$ otherwise.

Let $u \in A C\left(\mathbb{R}_{+}\right), y_{0} \in \mathbb{R}$ and denote $u^{*}:=\Omega(y, u)$. First, we would prove that for almost all $t \in \mathbb{R}_{+}$, $\dot{H}_{1}\left(\left(\Phi\left(u, y_{0}\right)\right)(t), u(t)\right)$ exists. Using (10) and with Leibniz derivative rule and denoting $y:=\left(\Phi\left(u, y_{0}\right)\right)$, we have

$$
\begin{aligned}
& \frac{\mathrm{d} H_{1}(y(t), u(t))}{\mathrm{d} t}=\dot{y}(t) u(t) \\
& +\left[\omega_{\Phi}\left(u^{*}(t), y(t), u(t)\right)-f_{a n}\left(u^{*}(t)\right)\right] \dot{u}^{*}(t) \\
& +\int_{u(t)}^{u^{*}(t)} \frac{\mathrm{d}}{\mathrm{d} t} \omega_{\Phi}(v, y(t), u(t)) \mathrm{d} v
\end{aligned}
$$

where we use the relation $\omega_{\Phi}(u(t), y(t), u(t))=y(t)$.

Let $t \geq 0$. The first term in the RHS of (A.1) exists for almost all $t \geq 0$ since $y(t)$ satisfies (1). Note that since $\omega_{\Phi}\left(u^{*}(t), y(t), u(t)\right)=f_{a n}\left(u^{*}(t)\right)$, the second term of (A.1) is zero since $\dot{u}^{*}(t)$ exists by the definition of $\Omega$. In order to get the dissipativity with the supply rate (2), it remains to check whether the last term of (A.1) exists, is finite and satisfies

$$
\int_{u(t)}^{u^{*}(t)} \frac{\mathrm{d}}{\mathrm{d} t} \omega_{\Phi}(v, y(t), u(t)) \mathrm{d} v \leq 0
$$

It suffices to show that, for every $v \in\left[u(t), u^{*}(t)\right]$, the following Dini's derivative :

$$
\lim _{\epsilon \searrow 0^{+}} \frac{1}{\epsilon}\left[\omega_{\Phi}(v, y(t+\epsilon), u(t+\epsilon))-\omega_{\Phi}(v, y(t), u(t))\right]
$$

exists and the limit is less or equal to zero when $u^{*}(t)>$ $u(t)$ and the limit is greater or equal to zero elsewhere.

For any $\epsilon \geq 0$, let us introduce the continuous function $\omega_{\epsilon}: \mathbb{R} \rightarrow \mathbb{R}$ by $\omega_{\epsilon}(v)=\omega_{\Phi}(v, y(t+\epsilon), u(t+\epsilon))$. More precisely, for every $\epsilon \geq 0, \omega_{\epsilon}$ is the unique solution of

$\omega_{\epsilon}(v)=\left\{\begin{array}{l}y(t+\epsilon)+\int_{u(t+\epsilon)}^{v} f_{1}\left(\omega_{\epsilon}(\sigma), \sigma\right) \mathrm{d} \sigma \forall v \geq u(t+\epsilon), \\ y(t+\epsilon)+\int_{u(t+\epsilon)}^{v} f_{2}\left(\omega_{\epsilon}(\sigma), \sigma\right) \mathrm{d} \sigma \forall v \leq u(t+\epsilon) .\end{array}\right.$

Note that $\omega_{0}(v)=\omega_{\Phi}(v, y(t), u(t))$ for all $v \in \mathbb{R}$ and

$$
\omega_{\epsilon}(u(t+\epsilon))=y(t+\epsilon) \quad \forall \epsilon \in \mathbb{R}_{+} .
$$

In order to show the existence of (A.3) and the validity of (A.2), we consider several cases depending on the sign of $\dot{u}(t)$ and $y(t)-f_{a n}(u(t))$.

First, we assume that $\dot{u}(t)>0$ that is not of measure zero. This implies that there exists a sufficiently small $\delta>0$ such that for every $\epsilon \in(0, \delta]$, we have $u(t+\epsilon)>u(t)$ and $\omega_{0}(u(t+\epsilon))=$ $y(t)+\int_{u(t)}^{u(t+\epsilon)} f_{1}\left(\omega_{0}(\sigma), \sigma\right) \mathrm{d} \sigma$. Moreover, with the change of integration variable $\sigma=u(\tau)$ we obtain $\omega_{0}(u(t+\epsilon))=y(t)+\int_{t}^{t+\epsilon} f_{1}\left(\omega_{0}(u(\tau)), u(\tau)\right) \dot{u}(\tau) \mathrm{d} \tau$, for all $\epsilon \in[0, \delta]$.

The functions $\epsilon \mapsto w_{0}(u(t+\epsilon))$ and $\epsilon \mapsto y(t+\epsilon)$ with $\epsilon \in(0, \delta]$ are two $A C$ functions which are solutions of the same locally Lipschitz ODE and with the same initial value. By uniqueness of solution, we get $\omega_{0}(u(t+\epsilon))=y(t+\epsilon)$. This fact together with (A.5) shows that $\omega_{\epsilon}(u(t+\epsilon))=\omega_{0}(u(t+\epsilon))$ for all $\epsilon \in[0, \delta]$. Let us evaluate (A.3) when $y(t) \geq f_{a n}(u(t))$. In this case, we have $u(t)<u^{*}(t)$ from the property of $\Omega$. Also, since for every $\epsilon \in(0, \delta]$ the two functions $\omega_{\epsilon}(v)$ and $\omega_{0}(v)$ satisfy the same ODE for $v \in\left[u(t+\epsilon), u^{*}(t)\right]$, we have $\omega_{\epsilon}(v)=\omega_{0}(v)$ for all $v \in\left[u(t+\epsilon), u^{*}(t)\right]$ and for all $\epsilon \in[0, \delta]$. This implies that $\lim _{\epsilon \searrow 0^{+}} \frac{1}{\epsilon}\left[\omega_{\epsilon}(v)-\omega_{0}(v)\right]=0$, for all $v \in\left[u(t), u^{*}(t)\right]$. Therefore, the inequality (A.2) holds for almost all $\dot{u}(t)>0$ and $y(t) \geq f_{\text {an }}(u(t))$.

Now, we check (A.2) when $y(t)<f_{a n}(u(t))$ and $\dot{u}(t)>0$ that is not of measure zero. Note that by the definition of $\Omega, u^{*}(t)<u(t)$ which implies that the integrand in (A.2) is evaluated for all $v \in\left(u^{*}(t), u(t)\right)$. Also, since $\dot{u}(t)>0$, there exists $\delta>0$ such that we have $v \leq u(t)<u(\tau)$ and $\dot{u}(\tau)>0$ for all $\tau \in(t, t+\gamma)$. It follows from (A.4) and assumption (A) that for every $\epsilon \in(0, \delta)$,

$$
\begin{aligned}
\frac{\mathrm{d} \omega_{\epsilon}(u(\tau))}{\mathrm{d} \tau} & =f_{2}\left(\omega_{\epsilon}(u(\tau)), u(\tau)\right) \dot{u}(\tau) \\
& \leq f_{1}\left(\omega_{\epsilon}(u(\tau)), u(\tau)\right) \dot{u}(\tau) \quad \forall \tau \in[t, t+\epsilon],
\end{aligned}
$$

and the function $y$ satisfies $\frac{\mathrm{d} y(\tau)}{\mathrm{d} \tau}=f_{1}(y(\tau), u(\tau)) \dot{u}(\tau)$ for all $\tau \in[t, t+\epsilon]$. Since $\omega_{\epsilon}(u(t+\epsilon))=y(t+\epsilon)$ and using the comparison principle (in reverse direction), we get that for every $\epsilon \in[0, \delta), \omega_{\epsilon}(u(\tau)) \geq y(\tau)$ for all $\tau \in[t, t+\epsilon]$. Since the two functions $\omega_{\epsilon}(v)$ and $\omega_{0}(v)$ for $v \in\left[u^{*}(t), u(t)\right]$ are two solutions of the same ODE, it follows that $\omega_{\epsilon}(v) \geq \omega_{0}(v)$ and we get that if it exists:

$$
\lim _{\epsilon \searrow 0^{+}} \frac{1}{\epsilon}\left[\omega_{\epsilon}(v)-\omega_{0}(v)\right] \geq 0 \quad \forall v \in\left[u^{*}(t), u(t)\right] .
$$

In the following, to show the existence of the limit given in (A.6), we compute a bound on the function $\epsilon \mapsto$ $\frac{1}{\epsilon}\left[\omega_{\epsilon}(v)-\omega_{0}(v)\right]$. Note that for every $\epsilon \in[0, \delta]$,

$$
\begin{aligned}
& \left|\omega_{\epsilon}(v)-\omega_{0}(v)\right| \leq|y(t+\epsilon)-y(t)|+\int_{u(t)}^{u(t+\epsilon)}\left|f_{2}\left(\omega_{\epsilon}(\sigma), \sigma\right)\right| \mathrm{d} \sigma \\
& +\int_{v}^{u(t)}\left|f_{2}\left(\omega_{\epsilon}(\sigma), \sigma\right)-f_{2}\left(\omega_{0}(\sigma), \sigma\right)\right| \mathrm{d} \sigma,
\end{aligned}
$$


for all $v \in\left[u^{*}(t), u(t)\right]$. By the locally Lipschitz property of $f_{2}$, by the boundedness of $f_{2}$ and by the boundedness of $\omega_{\epsilon}$ on $[v, u(t)]$ for all $\epsilon \in[0, \delta]$, we obtain

$$
\begin{aligned}
& \left|\omega_{\epsilon}(v)-\omega_{0}(v)\right| \leq|y(t+\epsilon)-y(t)| \\
& \quad+\int_{v}^{u(t)} L\left|\omega_{\epsilon}(\sigma)-\omega_{0}(\sigma)\right| \mathrm{d} \sigma+\alpha|u(t+\epsilon)-u(t)|,
\end{aligned}
$$

where $\alpha$ is a bound of $f_{2}$ on a compact set and $L$ is the Lipschitz constant of $f_{2}$ on $\left[\omega_{\min }, \omega_{\max }\right] \times[v, u(t)]$ with $\omega_{\min }:=\min _{(\epsilon, \sigma) \in[0, \delta] \times[v, u(t)]} \omega_{\epsilon}(\sigma)$ and $\omega_{\max }:=$ $\max _{(\epsilon, \sigma) \in[0, \delta] \times[v, u(t)]} \omega_{\epsilon}(\sigma)$. With the Gronwall's lemma [13], this implies that for every $\epsilon \in[0, \delta], \mid \omega_{\epsilon}(v)-$ $\omega_{0}(v) \mid \leq \exp ((u(t)-v) L)[|y(t+\epsilon)-y(t)|+\alpha \mid u(t+\epsilon)-$ $u(t) \mid]$ for all $v \in\left[u^{*}(t), u(t)\right]$. Hence $\lim _{\epsilon \searrow 0^{+}} \frac{1}{\epsilon} \mid \omega_{\epsilon}(v)-$ $\omega_{0}(v) \mid \leq \exp ((u(t)-v) L)\left[\left|f_{1}(y(t), u(t))\right|+\alpha\right] \dot{u}(t)$ for all $v \in\left[u^{*}(t), u(t)\right]$. Consequently the limit given in (A.6) exists. It implies that the inequality (A.2) holds for almost all $\dot{u}(t)>0$ and $y(t)<f_{a n}(u(t))$.

We can use similar arguments to prove that (A.2) is satisfied for almost all $\dot{u}(t)<0$.

Finally, when $\dot{u}(t)=0$, we simply get $\lim _{\epsilon \backslash 0^{+}} \frac{1}{\epsilon} \mid \omega_{\epsilon}(v)-$ $\omega_{0}(v) \mid=0$, by the continuity of the above bound.

To prove the last claim, we need to show that $H_{1}$ is nonnegative.

It is assumed in the hypotheses that $f_{1}$ is positive. If $u(t) \geq 0$ and $y(t) \geq f_{a n}(u(t))$, we have that $f_{a n}(v) \leq$ $y(t)$ for all $v \in[0, u(t)]$ and $f_{a n}(v) \leq \omega_{\Phi}(v, y(t), u(t))$ for all $v \in[u(t), \Omega(y(t), u(t))]$ by the definiton of $\Omega$. Hence

$$
\begin{aligned}
& H_{1}(y(t), u(t))=\int_{0}^{u(t)} y(t)-f_{a n}(v) \mathrm{d} v \\
& \quad+\int_{u(t)}^{\Omega(y(t), u(t))} \omega_{\Phi}(v, y(t), u(t))-f_{a n}(v) \mathrm{d} v \geq 0 .
\end{aligned}
$$

On the other hand, if $u(t)<0$ and $y(t) \geq f_{a n}(u(t))$, we have that $y(t) \leq \omega_{\Phi}(v, y(t), u(t))$ for all $v \in[u(t), 0]$ (due to the positivity of $f_{1}$ ). Also, by the definition of $\Omega$, $\Omega(y(t), u(t)) \geq 0$ implies that $f_{a n}(v) \leq \omega_{\Phi}(v, y(t), u(t))$ for all $v \in[0, \Omega(y(t), u(t))]$. Similarly, $\Omega(y(t), u(t))<$ 0 implies that $f_{a n}(v)>\omega_{\Phi}(v, y(t), u(t))$ for all $v \in$ $[\Omega(y(t), u(t)), 0]$. Hence

$$
\begin{aligned}
& H_{1}(y(t), u(t))=-\int_{u(t)}^{0} y(t)-\omega_{\Phi}(v, y(t), u(t)) \mathrm{d} v \\
& \quad+\int_{0}^{\Omega(y(t), u(t))} \omega_{\Phi}(v, y(t), u(t))-f_{a n}(v) \mathrm{d} v \geq 0 .
\end{aligned}
$$

For the case $y(t)<f_{a n}(u(t))$, the non-negativity of $H_{1}$ can be checked using the same routine and using the positivity of $f_{2}$.

\section{References}

[1] D. Angeli, "Systems with counterclockwise input-output dynamics," IEEE Trans. Aut. Contr., Vol. 51, no. 7, pp. 1130-1143, 2006.

[2] P.J. van Bree, C.M.M. van Lierop \& P.P.J. van den Bosch, "Control-oriented hysteresis models for magnetic electron lenses," IEEE Trans. Magnet., vol. 45, no. 11, pp. 5235-5238, 2009.
[3] M. Brokate \& J. Sprekels, Hysteresis and Phase Transitions, Springer Verlag, New York, 1996.

[4] B. Coleman \& M. Hodgdon, "On a class of constitutive relations for ferromagnetic hysteresis," Arch. Rat. Mech. Analys., vol. 99, no. 4, pp. 375-396, 1987.

[5] R.B. Gorbet, K.A. Morris, D.W.L. Wang, "Passivity-based stability and control of hysteresis in smart actuators," IEEE Trans. Contr. Syst. Techn., vol. 9, no. 1, pp. 5-16, 2001.

[6] P. Hartman, Ordinary Differential Equations. Second Edition, Birkhauser, 1982.

[7] R.V. Iyer, X. Tan, P.S. Krishnaprasad, "Approximate inversion of the Preisach hysteresis operator with application to control of smart actuators," IEEE Trans. Aut. Contr., vol. 50, pp. 798-810, 2005.

[8] F. Ikhouane, J. Rodellar, "A linear controller for hysteretic systems," IEEE Trans. Automat. Contr., vol. 51, pp. 340$344,2006$.

[9] B. Jayawardhana, R. Ouyang, V. Andrieu, "Dissipativity of general Duhem hysteresis models," Proc. 50th IEEE Conf. Dec. Contr. and Eur. Contr. Conf, Orlando, 2011.

[10] B. Jayawardhana, E.P. Ryan, A.R. Teel, "Bounded-energyinput convergent-state property of dissipative nonlinear systems: an iISS approach," IEEE Trans. Automatic Contr., vol. 55, no. 1, pp. 159-164, January 2010.

[11] B. Jayawardhana, V. Andrieu, "Sufficient conditions for dissipativity on Duhem hysteresis model," Proc. 48th IEEE Conf. Dec. Contr., Shanghai, 2009.

[12] D. C. Jiles, D. L. Atherton, Theory of ferromagnetic hysteresis, Journal of magnetism and magnetic material, vol. 61, no. 1-2, pp. 48-6, 1986.

[13] H.K. Khalil. Nonlinear Systems, 3rd edition, Prentice-Hall, Upper Saddle River, NJ, 2002.

[14] A. Lanzon, I.R. Petersen, "Stability robustness of a feedback interconnection of systems with negative imaginary frequency response," IEEE Trans. Aut. Contr., vol. 53, pp. 1042-1046, 2008.

[15] J.W. Macki, P. Nistri, P. Zecca, "Mathematical models for hysteresis," SIAM Review, vol. 35, no. 1, pp. 94-123, 1993.

[16] J. Oh, D.S. Bernstein, "Semilinear Duhem model for rateindependent and rate-dependent hysteresis," IEEE Trans. Automat. Contr., vol. 50, pp. 631-645, 2005.

[17] R. Ortega, A. Loría, P.J. Nicklasson, H. Sira-Ramírez, Passivity-Based Control of Euler-Lagrange Systems, Springer-Verlag, London, 1998.

[18] A.K. Padthe, J-H. Oh, D.S. Bernstein, "Counterclockwise Dynamics of a Rate-independent Semilinear Duhem Model," Proc. 44th IEEE Conf. Dec. Contr. and Eur. Contr. Conf., Seville, 2005

[19] A.J. van der Schaft, $L_{2}$-Gain and Passivity Techniques in Nonlinear Control, Springer-Verlag, London, 2000.

[20] J. Takacs, "Mathematical proof of the definition of anhysteretic state", Physical B, vol. 372, pp. 57-60, 2006.

[21] H.L. Trentelmann, J.C. Willems, "Every storage functions is a state function," Systems and Control Letters, vol. 32, pp. 249-259, 1997

[22] A. Visintin, Differential Models of Hysteresis, SpringerVerlag, New York, 1994.

[23] C.C. de Wit, H. Olsson, K.J. Astrom, P. Lischinsky, "A new model for control of systems with friction," IEEE Trans. Aut. Contr., vol. 40, no. 3, pp. 419-425, 1995. 